\title{
WIDE FIELD SPECTROSCOPY AND THE UNIVERSE
}

\author{
MATTHEW COLLESS \\ Mount Stromlo 83 Siding Spring Observatories \\ The Australian National University \\ Canberra, ACT, Australia
}

\section{Introduction}

In this short survey of the applications of wide-field, multi-object spectroscopy to galaxy evolution, large-scale structure and cosmology, I interleave summaries of the general goals and state of play in these fields with specific examples based on my own recent work. I first briefly review the goals and figures of merit for current and future redshift surveys, before examining some recent results from deep surveys in the field, cluster redshift surveys and surveys of large scale structure. I take a look beyond redshift surveys to other probes of galaxy evolution before concluding with a discussion of the future prospects for such studies using the next generation of wide-field, multi-object spectrographs on large telescopes.

The main goals of extragalactic/cosmological studies for which widefield, multi-object spectroscopy is relevant are (i) the world model: $H_{0}, q_{0}$, $\Omega, \Lambda$, etc.; (ii) large-scale structure: $P(k), \xi(r)$, Gaussianity, topology, morphology, biassing, etc.; and (iii) galaxy formation and evolution: luminosity function and $\xi(r)$ as functions of galaxy type, environment and lookback time, the redshift of peak star formation in bulges and disks, etc. Redshift surveys are essential to some of these topics and one powerful alternative in studying others, either because they are intrinsically population studies or because they demand large statistical samples. In either case one is playing a numbers game, so that the most efficient observational approach will employ wide-field multi-object spectroscopy.

\section{Current Results from Redshift Surveys}

There has been much recent progress in studies of galaxy formation and evolution. The general form of the evolution of the galaxy population out 
to $z \sim 1$ has begun to emerge from deep redshift surveys of $\sim 10^{3}$ field galaxies to $B=24, I=22$ and $K=20$ (Ellis et al. 1996, Lilly et al. 1995, Cowie et al. 1996). These studies have revealed differential evolution of the galaxy luminosity function with luminosity and also with colour or spectral type. At the same time, significant advances have been made in detecting highredshift $(z \gtrsim 3)$ star-forming galaxies directly from their stellar light and not from an associated QSO or radio source (Steidel et al. 1996).

At the other end of the scale, several redshift surveys of $\sim 10^{4}$ nearby $(z \lesssim 0.1)$ galaxies have been carried out over large volumes of the local universe in order to map large-scale structure (CfA, SSRS, IRAS PSCz, LCRS, ORS, etc.). These surveys have measured the galaxy distribution's power spectrum, $P(k)$, or correlation function, $\xi(r)$, with fair precision on scales less than about $100 \mathrm{~h}^{-1} \mathrm{Mpc}$, but also reveal still larger structures, suggesting that we have not yet mapped a sufficiently large volume to constitute a fair sample of the universe.

Studies measuring both the redshift and the distance for $\sim 10^{3}$ galaxies at redshifts $c z \lesssim 5000 \mathrm{~km} \mathrm{~s}^{-1}$ have mapped the peculiar motions in the local universe and thereby constrained the local mass distribution and the nature of the missing mass. Such observations are much more difficult than simple redshift surveys, as the usual distance indicators are based on versions of the linewidth-luminosity relation (the Fundamental Plane for ellipticals, the Tully-Fisher relation for spirals) and so require measurements of the internal motions of each galaxy. As discussed below, such studies are beginning to be pushed to higher redshifts in order to use the linewidthluminosity relations as probes of galaxy evolution.

The use of galaxy redshift surveys to probe the parameters of the world model, popular twenty years ago, has largely been abandoned today due to the realisation that evolutionary effects on the galaxy population swamp the more subtle effects of spacetime geometry.

There are at least two other important types of redshift survey which I do not touch on at all here, namely surveys of QSOs (to probe their evolution and very large-scale structures) and surveys of QSO absorption line systems (ALS) and their associated galaxies (which offer an alternative approach to studying galaxy evolution at high redshift).

\section{Figures of Merit for Surveys}

Table 1 gives a brief summary of some recent and proposed redshift surveys, along with the diameter of the telescope and the field of view and multiplex

of the spectrograph with which they are carried out. The numbers are in some cases merely indicative, especially for future surveys.

Various figures-of-merit can be envisaged for wide-field, multi-object 
TABLE 1. Summary of some recent and proposed redshift surveys.

\begin{tabular}{lrccccrr}
\hline Survey & $N_{\text {gal }}$ & Band & $\begin{array}{c}\text { Mag } \\
\text { lim }\end{array}$ & $\bar{z}$ & $\begin{array}{c}\text { Diam. } \\
(\mathrm{m})\end{array}$ & $\begin{array}{r}\text { FoV } \\
\left(\square^{\circ}\right)\end{array}$ & $\begin{array}{r}\text { Multi } \\
\text {-plex }\end{array}$ \\
\hline CfA & 15000 & $B$ & 15.5 & 0.02 & 1.5 & - & 1 \\
SSRS & 10000 & $B$ & 15.5 & 0.02 & 1.5 & - & 1 \\
SAPM & 1800 & $b_{J}$ & 17.2 & 0.04 & 2.3 & - & 1 \\
PSCz & 15000 & $S_{60}$ & 0.6 & 0.03 & 2.1 & - & 1 \\
LCRS & 26000 & $R$ & 17.7 & 0.10 & 2.5 & 1.50 & 112 \\
ESP & 4000 & $b_{J}$ & 19.4 & 0.10 & 3.6 & 0.50 & 50 \\
ESOSc & 1000 & $R$ & 20.5 & 0.30 & 3.6 & 0.70 & 50 \\
Autofib & 1400 & $b_{J}$ & 22.5 & 0.30 & 3.9 & 0.70 & 64 \\
CFRS & 760 & $I$ & 22.5 & 0.56 & 3.6 & 0.02 & 70 \\
ORS & 8500 & $B$ & 14.5 & 0.01 & 1.5 & - & 1 \\
MRSP & 750000 & $b_{J}$ & 20.5 & 0.15 & 1.2 & 30.00 & 15000 \\
2dFb & 250000 & $b_{J}$ & 19.7 & 0.10 & 3.9 & 2.10 & 400 \\
2dFf & 6000 & $r_{F}$ & 21.0 & 0.30 & 3.9 & 2.10 & 400 \\
SDSS & 1000000 & $g$ & 18.3 & 0.10 & 2.5 & 3.00 & 640 \\
FLAIR & 100000 & $J$ & 13.0 & 0.04 & 1.2 & 30.00 & 150 \\
VLTIR & 100000 & $K$ & 21.0 & 1.00 & 8.2 & 0.35 & 200 \\
LAMOST & 10000000 & $B$ & 20.0 & 0.12 & 4.0 & 21.20 & 5000 \\
\hline
\end{tabular}

Notes (undated references are to these proceedings): CfA = Center for Astrophysics redshift survey (Kurtz, da Costa); SSRS = Southern Sky Redshift Survey (da Costa); SAPM $=$ Stromlo-APM redshift survey (Loveday et al. 1992); PSCz = IRAS Point Source Catalogue $z$-survey (Rowan-Robinson); LCRS = Las Campanas Redshift Survey (Shectman et al. 1996, preprint); ESP = ESO Slice Project (Vettolani, Zucca); ESOSc = ESOSculptor faint galaxy redshift survey (Bellanger et al. 1995); Autofib = Autofib redshift survey (Ellis et al. 1996, this paper); CFRS = Canada France Redshift Survey (Lilly et al. 1995, Hammer); ORS = Optical Redshift Survey (Santiago et al. 1995); MRSP = Münster Redshift Survey Project (Schuecker); $2 \mathrm{dFb} / 2 \mathrm{dFf}=2 \mathrm{dF}$ bright and faint redshift surveys (this paper); SDSS = Sloan Digital Sky Survey (Kron); FLAIR = FLAIR southern sky survey (Parker et al.); VLTIR = VLT near-IR redshift survey (this paper, Le Fevre); LAMOST = Large Area Multi-Object Spectroscopy Telescope (Chu).

spectrographs. The usual figure-of-merit for imaging surveys is $A \Omega$ : the product of the telescope aperture $A$ and the imaging field-of-view $\Omega$. The analogous figure for spectroscopic surveys is $A M$ : the product of the telescope aperture and the multiplex $M$ of the spectrograph (i.e. the number of objects which can be observed simultaneously). Note in the following exercise we are not comparing the redshift surveys with each other (in which case a more appropriate figure-of-merit might be the effective volume surveyed), but rather the potential of a given telescope/spectrograph combination for carrying out such surveys.

Figure 1a shows $A M$ as a function of sample size for the surveys listed in 

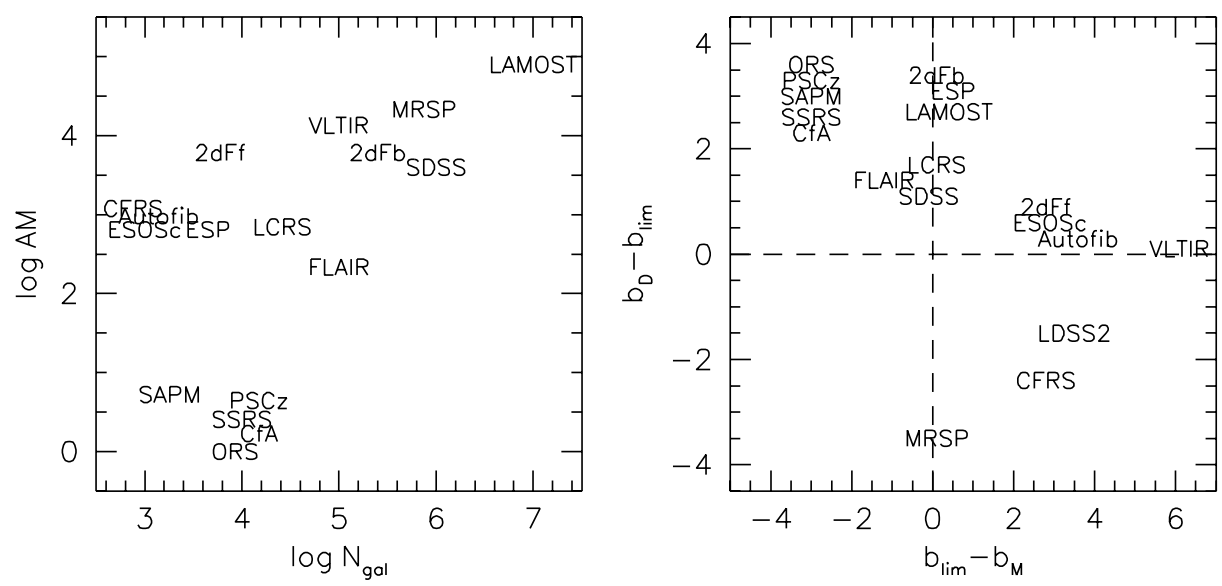

Figure 1. (a) The product of telescope aperture, $A$, and spectrograph multiplex, $M$, versus the survey size, $N_{g a l}$, for various current and future galaxy redshift surveys. (b) The distribution of survey magnitude limits, $b_{l i m}$, compared to the limiting magnitude of the telescope for spectroscopy, $b_{D}$, and the magnitude where the number of galaxies in the field equals the spectrograph multiplex, $b_{M}$.

Table 1. The one-by-one surveys (Stromlo-APM, CfA, SSRS, ORS, PSCz) all have $\log A M<1$ since they were all carried out on small $(1 \mathrm{~m}-2 \mathrm{~m})$ telescopes, yet they have sample sizes comparable to any of the recent group of redshift surveys carried out on larger $(2 \mathrm{~m}-4 \mathrm{~m})$ telescopes with multiobject spectrographs. This reflects partly the large amount of time spent by dedicated observers, but, more importantly, the small field sizes available on larger telescopes. This constraint has meant that (with the exception of LCRS) most recent multi-object redshift surveys have concentrated on going deep rather than covering a large volume. As the figure shows, this situation is expected to change in the near future, as large surveys are begun with spectrograph/telescope combinations with larger $A M$ (FLAIR on the $1.2 \mathrm{~m}$ UKST with 92 fibres and $6^{\circ} \mathrm{FoV}$; SDSS on a $2.5 \mathrm{~m}$ with 640 fibres and $3^{\circ} \mathrm{FoV}$; and the $2 \mathrm{dF}$ redshift survey on a $4 \mathrm{~m}$ with 400 fibres and $\left.2^{\circ} \mathrm{FoV}\right)$. These instruments will increase sample sizes by an order of magnitude for both bright surveys (over $10^{5}$ galaxies with $B=17-19.5$ for FLAIR, SDSS and the $2 \mathrm{dF}$ bright survey) and faint surveys $\left(\sim 10^{4}\right.$ galaxies with $R=21$ for the $2 \mathrm{dF}$ faint survey). In the more distant future something like the LAMOST concept (Chu, these proceedings) will achieve another order of magnitude increase in $A M$, while multi-object spectrographs on $8 \mathrm{~m}$-class telescopes will perform surveys of $10^{5}$ galaxies at yet fainter limits.

As well as $A M$, there are a number of other secondary indicators of capability. One of these is the range of limiting magnitudes, $b_{M} \lesssim b_{\text {lim }} \lesssim$ $b_{D}$, over which a particular telescope/spectrograph is an effective survey 
tool. Here $b_{M}$ is defined by $N\left(<b_{M}\right)=M / \Omega$ (i.e. the magnitude at which the instrument is well-matched to the surface density of galaxies), while $b_{D} \approx 23+5 \log (D / 4)$ is the approximate limiting magnitude achieved in one half-night on a telescope of diameter $D$ (a psychological rather than physical limit, but an effective one nonetheless). Figure $1 \mathrm{~b}$ shows how the magnitude limits are chosen within this range. 'Big' surveys, for which the prime goal is sample size, have $b_{l i m} \approx b_{M}$, while 'deep' surveys, for which the prime goal is sample depth, have $b_{\text {lim }} \approx b_{D}$. The single-object surveys, for which $b_{l i m}-b_{M}$ is arbitrarily set to -3 , still have $b_{D}-b_{\text {lim }}$ comparable to other 'big' surveys. Two ultra-deep surveys (LDSS2 and CFRS) have $b_{D} \ll b_{\text {lim }}$, as does the MRSP objective prism survey (where extra depth is obtained at the cost of resolution).

The other indicators of capability are the accessible wavelength range, the spectral resolution $(R \equiv \lambda / \Delta \lambda)$, and the instrumental throughput (which determines the $S / N$ achieved in fixed time). For redshift surveys we want the largest possible spectral range but modest $R(\lesssim 500$ for evolution, $\lesssim 1000$ for structure) and S/N $(<10)$.

\section{Redshift Surveys for Galaxy Evolution}

For redshift surveys investigating galaxy evolution, the ultimate aims are to uncover the important physical processes in forming galaxies, what makes galaxies biased tracers of the mass, and what part environment plays in galaxy evolution. The more immediate aims are to fully characterise the distributions of galaxy properties (luminosity, mass, star-formation rate, type, etc.), to determine how these properties depend on environment (i.e. local structure and over-density), and to recover the evolution of the galaxy population with redshift.

Results come from two types of survey:

1. $b_{\text {lim }} \sim b_{M}$ surveys of $\sim 10^{4}$ galaxies at $z \sim 0$ (e.g. LCRS and PSCz) with the goal of definitively establishing the properties of the local galaxy population. The existing surveys are, however, too small to fully explore type/environment dependencies; proposed surveys of $10^{5}-10^{6}$ galaxies (e.g. $2 \mathrm{dFb}$ and SDSS) will overcome this limitation. For such surveys good photometry and uniform selection criteria are crucial.

2. $b_{\text {lim }} \sim b_{D}$ surveys of $\sim 10^{3}$ galaxies at $z \approx 0.5-1$ (e.g. CFRS and Autofib) with the goal of tracking the evolution of the galaxy population. Wide-area deep imaging is a prerequisite for such surveys. The $2 \mathrm{dF}$ faint redshift survey will cover $\sim 10^{4}$ galaxies selected from deep photographic imaging, but the next generation of surveys on $8 \mathrm{~m}$-class telescopes will demand wide-area CCD imaging. In order to push beyond $z \sim 1$ will require a near-infrared multi-object spectrograph (see below; 
also Le Fevre, these proceedings), as the familiar optical lines are redshifted beyond $1 \mu \mathrm{m}$ and the spectral 'desert' of the restframe UV is shifted into the optical. Beyond $z \sim 3$, optical spectroscopy is appropriate again as $\operatorname{Ly} \alpha$, CIV and the Lyman limit become accessible.

\section{The Autofib Redshift Survey}

The Autofib redshift survey (Ellis et al. 1996, Heyl et al. 1996), along with the CFRS (Lilly et al. 1995), defines the current state of deep field surveys. The main motivation for the survey was tracking the evolution of the galaxy luminosity function (LF) out to $z \sim 0.5$ over a wide range of intrinsic luminosities. A survey with broad coverage in both $L$ and $z$ implies the sample must span a very broad range of apparent magnitudes. Our solution was to combine several of our previous redshift surveys, with magnitude limits between $B=17$ and $B=24$, with $\sim 1000$ new galaxy redshifts filling the gaps between these surveys and increasing the total sample size.

The local LF that we obtain from the 291 galaxies with $0.02<z<0.1$ is adequately represented by a Schechter function over the range $-20<M<$ -14 . The best-fit parameters are: $M^{*}=-19.3+5 \log h, \phi^{*}=0.026 h^{3} \mathrm{Mpc}^{-3}$, $\alpha=-1.1$. This fit is compared in Figure 2 to similar determinations of the local LF obtained in other studies. The significant differences between these results are not understood. Specific problems with some individual surveys have been noted (e.g. clustering in the DARS survey, Zwicky magnitudes and the presence of Virgo in the CfA survey), while the discrepancies between the other surveys have variously been ascribed to magnitude scale errors, different isophotal magnitude systems, ultra-large scale structure, and very rapid evolution at low redshift. None of these possibilities are very palatable, nor particularly credible. The solution to this problem is not bigger redshift surveys (except that these will tie down the faint end of the LF somewhat more securely) but rather better samples, with lowisophote photometry of uniform quality and calibration over large areas, and selection criteria that are as inclusive as possible (particularly with respect to low surface brightness galaxies).

The broad magnitude range and faint limiting magnitude of the combined Autofib redshift survey gives unambiguous evidence for LF evolution with redshift. The faint end of the overall LF becomes progressively steeper with increasing lookback time (see Figure 10 of Ellis et al. 1996), so that by $z \sim 0.5$ there is a noticeable change in the LF around $L^{*}$. A $\chi^{2}$ test rejects a non-evolving LF with a probability $<10^{-20}$. Although evolution is clearly significant for $z \gtrsim 0.3$, whether there is evolution at $z \lesssim 0.3$ remains uncertain due to the uncertainties in the local LF. The change in the LF shape is due to the rapid evolution of star-forming galaxies with high [OII] 


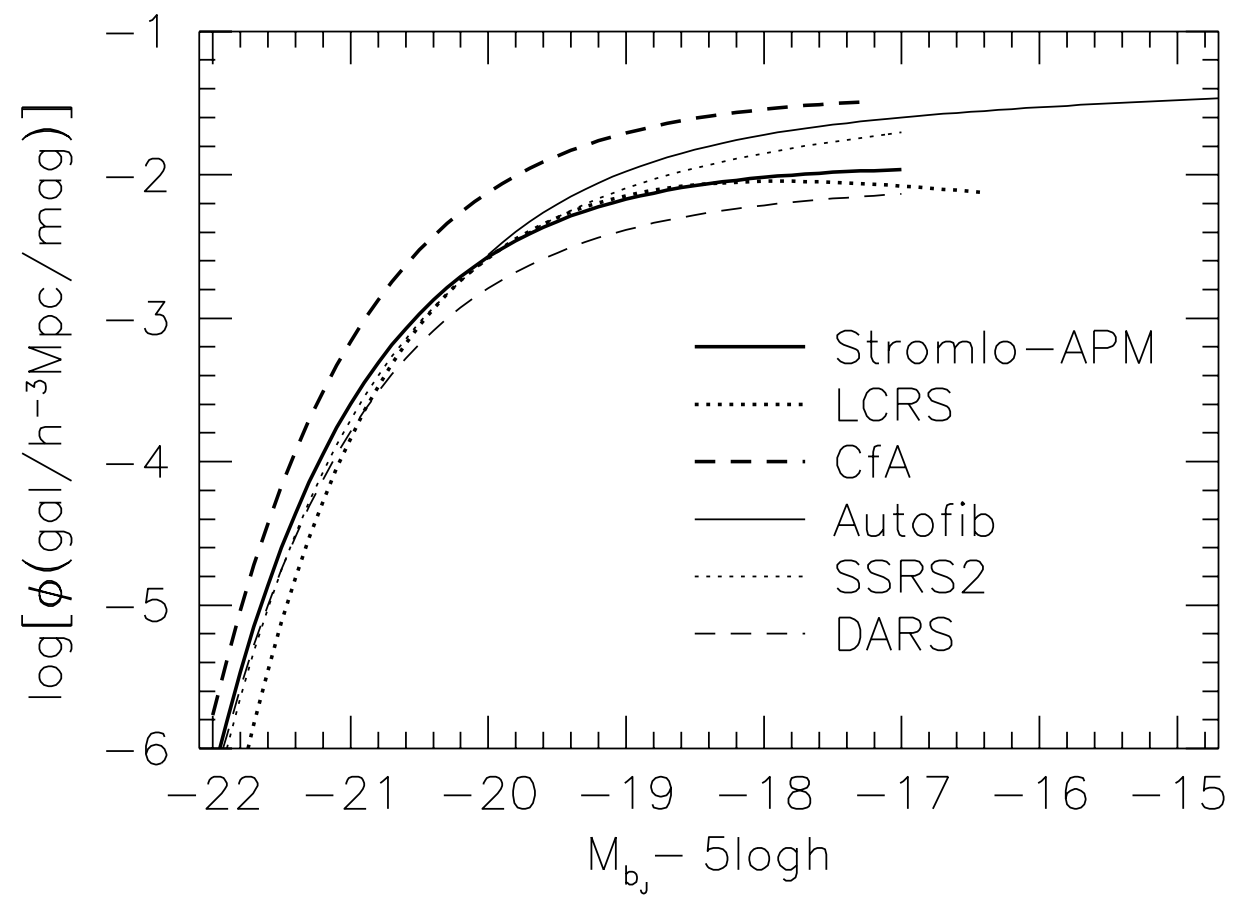

Figure 2. A comparison of the Schechter function fits to various determinations of the local galaxy luminosity function. We put all the luminosity functions on the $b_{J}$ system, assuming $b-r=1.1$ for LCRS and $b-b_{Z \text { wicky }}=-0.7$ for CfA (to give the same $M^{*}$ as the others). See caption to Table 1 for survey references; DARS is Efstathiou et al. (1988).

equivalent widths. Figure 3 shows that the median equivalent width of [OII] amongst late-type spiral galaxies increases with redshift at fixed luminosity, implying higher rates of star-formation in all objects, with bright galaxies showing this increase at higher redshift than faint galaxies. The evolution of the LF for each spectral type (see Heyl et al. 1996) reflects this effect: the E/S0 LF shows no significant evolution out to at $z \sim 0.7$; the early-spiral LF shows a steepening of the faint-end slope, though the number of $L^{*}$ galaxies does not change by $z \sim 0.5$; the late-spiral LF evolves strongly, with even $L^{*}$ galaxies increasing in number by $z \sim 0.5$. We conclude that the evolution of the population to $z \sim 0.5$ is completely dominated by late types.

\section{Cluster Redshift Surveys}

Current redshift surveys in clusters are focussed on cluster structure and dynamics or the evolution of galaxies in clusters. There is a clear linkage between these topics, since the detailed dynamical analysis of cluster substructure can reveal the formation history of the cluster, and hence the 


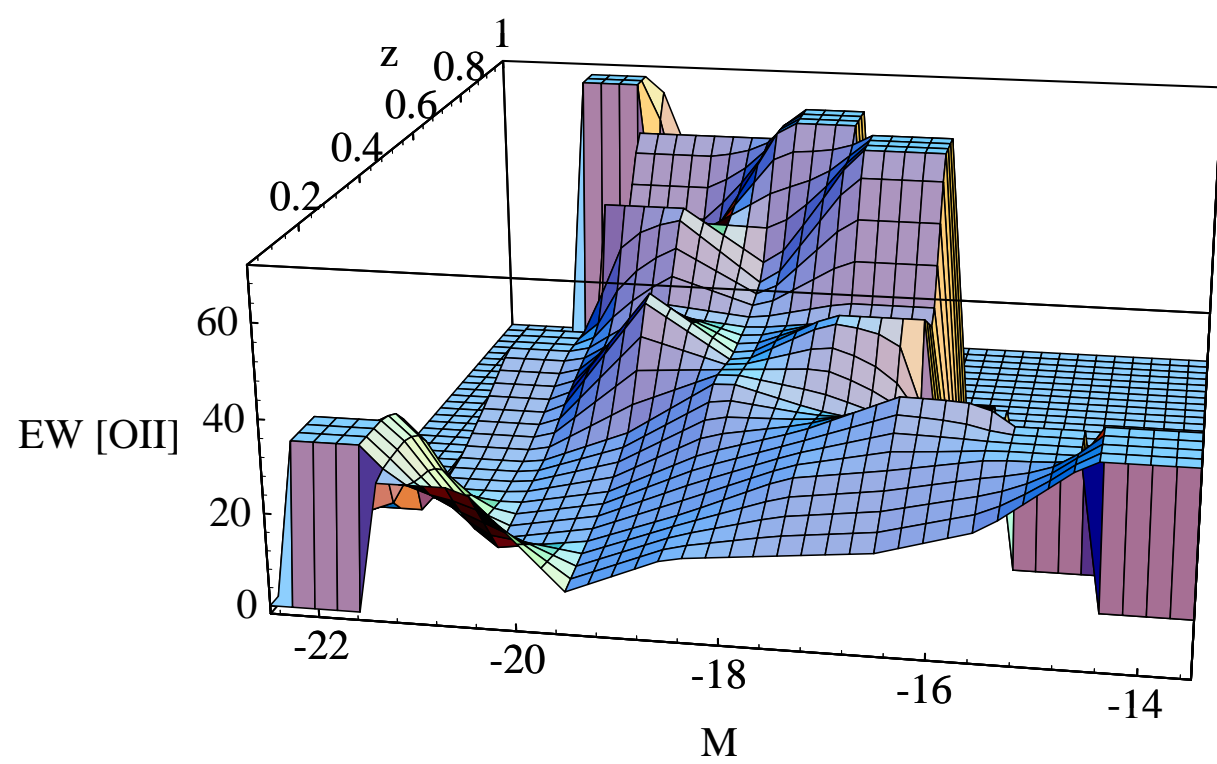

Figure 3. The median [OII] equivalent width (an indicator of star-formation rate) as function of luminosity and redshift for the late-type spirals in the Autofib survey.

environmental effects suffered by the individual galaxies. Another motivation for some recent cluster surveys (e.g. the CNOC survey; Carlberg et al. 1996) is to map the infall pattern of galaxies around clusters, which can be used to estimate $\Omega_{\text {cluster }}$.

Again, various types of survey are appropriate depending on the specific goals. Complete redshift surveys of single clusters to faint limits $\left(\sim 10^{3}\right.$ galaxies) can yield the detailed formation history of the cluster and the dynamics of the galaxies as a function of type and luminosity. By contrast, surveys of $\sim 10^{2}$ galaxies in each of $\sim 10^{2}$ clusters allow one to study cluster dynamics and populations as function of richness/overdensity. They can also be used (as in the CNOC survey) to average over structures and obtain $\langle\Omega\rangle$ from infall patterns at large radii. Finally, deep surveys of distant clusters yield the evolution of populations and dynamics.

\section{The Merger History of the Coma Cluster}

By measuring 243 new redshifts in Coma to obtain a sample of of 465 cluster members with velocities, Colless \& Dunn (1996) were able to recover the merger history of the cluster from kinematic evidence. The line-of-sight velocity distribution in Coma proves to be highly non-Gaussian, which a new test for localised velocity structure shows is caused by the existence 
of a dynamically distinct group around NGC 4839. A maximum likelihood mixture model yields a robust partition of the sample into a main cluster around NGC 4874 and this NGC 4839 group, which accounts for about $10 \%$ of the total mass. A linear two-body orbit model indicates that the two clusters are at $74^{\circ}$ to the line of sight with a separation of $0.8 \mathrm{~h}^{-1} \mathrm{Mpc}$, and are approaching each other at $1700 \mathrm{~km} \mathrm{~s}^{-1}$. Velocity maps of the cluster core show that there is an ongoing merger between the main cluster and another group around NGC 4889.

Several lines of evidence, including the substructure revealed in velocity space, the distribution of X-ray gas and lack of a cooling flow, the diffuse radio halo, and the $\mathrm{cD}$ envelopes around NGC 4874 and NGC 4839 but not NGC 4889, together imply a self-consistent merger history for Coma. In this picture, the NGC 4839 group is falling into Coma along the Great Wall from the direction of A1367 and is just beginning to penetrate the main cluster. The NGC 4889 group is partially disrupted due to its ongoing merger with the main cluster and has ejected NGC 4889, once its dominant galaxy. NGC 4874 is the original dominant galaxy of the main Coma cluster, now slightly dislodged from the bottom of the potential well by its recent encounter with NGC 4889.

\section{Large Scale Structure Redshift Surveys}

Redshift surveys investigating large scale structure have as their main aims the characterisation of the structure (via the power spectrum or correlation function and measures of the Gaussianity, topology and morphology of the galaxy distribution) and the determination of the overall mass density $\Omega$ and the biasing parameter for galaxies $b$.

Specific goals in deriving the power spectrum $P(k)$ (or, equivalently, the correlation function $\xi(r))$ are to link the small-scale, $z \sim 0$ galaxy $P(k)$ to the much larger-scale, high- $z P(k)$ obtained from the microwave background by COBE and other anisotropy experiments. Defining the shape of $P(k)$ gives the initial mass fluctuation spectrum, yielding clues ot both the nature of the dark matter and the value of $\Omega$. Current surveys give $P(k)$ to a precision

of $\sim 20 \%$ at $30 \mathrm{~h}^{-1} \mathrm{Mpc}$, and to within a factor of 2 at $100 \mathrm{~h}^{-1} \mathrm{Mpc}$. The expected turnover in $P(k)$ at somewhat larger scales is only hinted at. Determining $P(k)$ accurately on these scales will require massive $\left(10^{5}-10^{6}\right)$ galaxy surveys at $z \sim 0.1$, while establishing the evolution of $P(k)$ at higher redshifts will require large $\left(10^{4}-10^{5}\right)$ surveys of galaxies at $z \sim 1$ and of QSOs at $z \gg 1$.

A crucial question is whether the large-scale (linear-regime) galaxy distribution implies an underlying Gaussian distribution of mass fluctuations, as predicted in many inflationary cosmologies. If so, the large-scale dis- 
tribution is fully described by its power spectrum. On smaller scales the question is whether the topology/morphology of the distribution is consistent with the formation of structure by gravitational instability within the framework of some model for the dark matter and the spectrum of initial fluctuations. This requires surveys of compact, densely-sampled volumes.

The mass density, $\Omega$, and bias parameter, $b$, are tightly coupled in the galaxy distribution, and the directly measurable quantity in most cases (e.g. from the redshift-space distortions in the distribution) is $\beta=\Omega^{0.6} / b$. A sufficiently large survey with galaxy type information would be able to use such methods to establish the relative biasing of the different galaxy types, a powerful constraint on theories of galaxy formation and evolution.

\section{The 2dF Galaxy Redshift Survey}

The main features of the $2 \mathrm{dF}$ spectrograph on the $3.9 \mathrm{~m}$ Anglo-Australian Telescope (see Figure 4) are: (i) a 2-degree field of view with atmospheric dispersion compensation, resulting from a new 4-component prime focus corrector; (ii) 400 fibres of 2 arcsec diameter controlled by a fast robot positioner with 0.2 arcsec precision; (iii) focal plane imaging using a CCD camera to assist in field acquisition and guiding; (iv) offline configuration in $<30$ min, with two focal planes on a tumbler mechanism eliminating deadtime for exposures longer than this; (v) twin spectrographs each taking 200 fibres and giving resolutions of $1.7 \AA$ to $8.3 \AA$ over $3500-10000 \AA$; (vi) pipeline reductions of data to return calibrated spectra to the observers in real-time. More details are given by Cannon (these proceedings).

Two major galaxy redshift surveys are being carried out with $2 \mathrm{dF}$ by a joint Anglo-Australian collaboration: a bright $\left(b_{J}<19.5\right)$ survey of 250,000 galaxies and a faint $(R<21)$ survey of 6000 galaxies. The overall goals of these surveys are to study large-scale structure and galaxy evolution, and especially the interrelation of structure and evolution. The bright survey limit of $b_{J}=19.5$ (extinction-corrected) implies a mean depth for the sample of $\bar{z}=0.1$. The total area of the survey is 1700 sq.deg., comprising a contiguous $75^{\circ} \times 12.5^{\circ}$ strip in the south Galactic cap, a contiguous $65^{\circ} \times 7.5^{\circ}$ strip in the north Galactic cap, and 100 random $2 \mathrm{dF}$ fields over the area of the southern APM galaxy survey. The 250,000 galaxies will be sampled over this area with almost $100 \%$ completeness. The faint survey of 6000 galaxies will be carried out in override mode, taking advantage of the highest-quality observing conditons occurring $10 \%$ of the time at the AAT.

We estimate that the total time required for the survey will be 90 nights. The survey will begin towards the end of 1996, and we hope to complete it by the end of 1998. Compared to the LCRS, the $2 \mathrm{dF}$ survey has the same

magnitude limit and redshift depth but $2.5 \times$ the area, $4 \times$ the sampling rate 


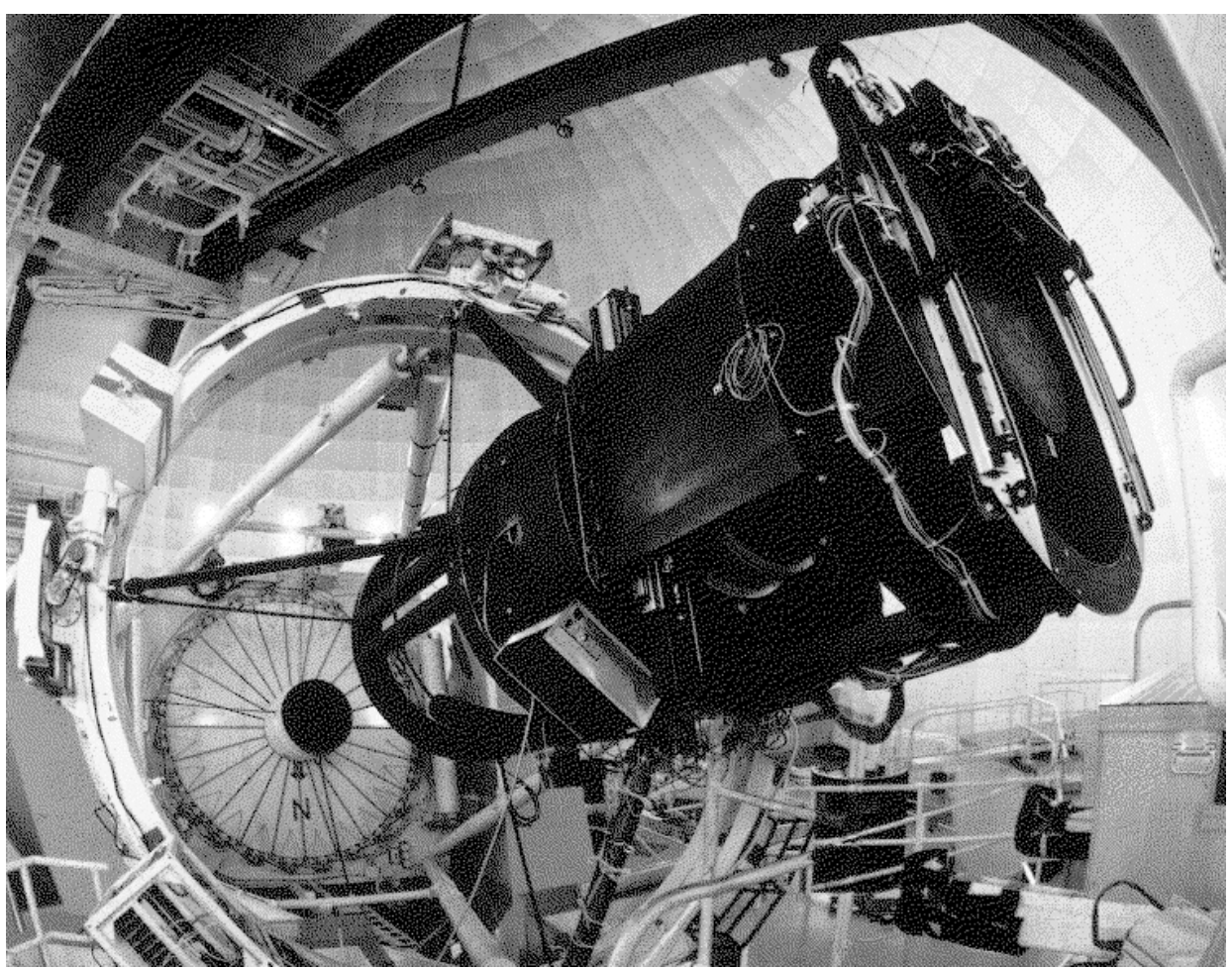

Figure 4. The $2 \mathrm{dF}$ fibre positioner and spectrograph mounted on the AAT. (Photo by Francisco Diego.)

and $10 \times$ the total number of galaxies. The $2 \mathrm{dF}$ survey will be complementary to the northern SDSS redshift survey, which has a similar magnitude limit but is planned to cover a larger area and include up to $10^{6}$ galaxies over it's five-year nominal lifetime (Kron, these proceedings).

Future redshift surveys with $2 \mathrm{dF}$ will take advantage of having such a large multiplex advantage on a $4 \mathrm{~m}$-class telescope to carry out massive surveys of faint objects. For example, investing a similar amount of time in the faint survey as the bright survey would yield 50,000 redshifts for galaxies to $R=21$, i.e. a survey twice as large as LCRS but with a mean redshift of 0.3 , giving a direct view of the evolution of galaxy clustering over the last third of the history of the universe.

\section{Beyond Redshift Surveys}

Redshift surveys generally use $\mathrm{R} \leqslant 1000$ and $\mathrm{S} / \mathrm{N} \lesssim 10$ in order to maximise the number of redshifts obtained per unit telescope time. However, large surveys with $\mathrm{R} \gtrsim 3000$ and $\mathrm{S} / \mathrm{N} \gtrsim 20$ are needed: (i) to measure the internal motions of 
galaxies to the faintest possible limits; (ii) to measure star-formation rates from $\mathrm{H} \alpha$ flux for large, representative samples; (iii) to investigate the starforming dwarf population (with narrow lines) at low to moderate redshift; (iv) to determine variations in the Fundamental Plane (FP) and TullyFisher (TF) relations as a function of luminosity, morphological type and environment; (v) to use the $\mathrm{FP} / \mathrm{TF}$ relations to map peculiar motions in the local universe; and (vi) to explore the evolution of the TF/FP relations as a constraint on galaxy evolution.

Few such surveys have yet been carried out, but some first steps are already being taken towards the latter goal. Van Dokkum \& Franx (1996) and Bender et al. (1996) have used the FP in clusters out to $z \sim 0.5$ to constrain the evolution of elliptical galaxies and obtain an estimate of $q_{0}$, while Rix et al. (1996) and Simard \& Pritchet (1996) have studied the evolution of the linewidth-luminosity relation for field galaxies in order to estimate the amount of luminosity evolution occurring in galaxies out to $z \sim 0.5$. At even higher redshifts, Vogt et al. (1996) have used the Keck telescope to measure rotation curves for a few galaxies out to $z \sim 1$. This exciting new probe of galaxy evolution is still being developed, and larger samples are clearly needed (at low redshift as well as high redshift) in order to fully understand the results hinted at in these first studies.

\section{Future Prospects for Survey Spectroscopy}

There are at least three main tracks for survey spectroscopy to take:

1. 4m-class aperture, very wide field $\left(\gtrsim 2^{\circ}\right)$, very large number of fibres ( $\gtrsim$ several hundred): optimal for undertaking massive $\left(\gtrsim 10^{6}\right.$ objects) surveys of galaxies/QSOs to $\mathrm{B} \approx 23$ and $z \lesssim 1$; this approach is currently exemplified by $2 \mathrm{dF}$, with the future perhaps looking like LAMOST (Schmidt field, 4m aperture, 5000 fibres; see Chu, these proceedings).

2. 8m-class aperture, small $\left(\leqslant 10^{\prime}\right)$ field, low multiplex $(<100)$, 'cheap' spectroscopic survey telescopes: optimal for carrying out surveys of faint, low-density sources (e.g. QSOs, ALS, radio galaxies); this approach is exemplified by the Hobby Eberley Telescope (Sebring et al. 1995).

3. 8m-class aperture, moderate $\left(\gtrsim 10^{\prime}\right)$ field, large $(\gtrsim 100)$ multiplex: optimal for massive surveys of very faint objects of high surface density such as high-redshift galaxies; exemplified by the DEIMOS optical multislit spectrograph proposed for the Keck telescope and by the optical/near-IR multi-object spectrographs proposed for the VLT (see below; also Le Fevre, these proceedings). 


\section{AUSTRALIS: a Multi-Object Spectrograph for the VLT}

AUSTRALIS is one of two concept studies for a multi-object optical/nearinfrared spectrograph optimised for high-redshift observations on ESO's VLT (the alternate VIRMOS concept is discussed by Le Fevre, these proceedings). A near-IR spectrograph is essential because for $z>1$ most of the familiar, strong and well-studied spectral features seen in the restframe visible have been shifted into the near-IR. A multi-object capability is likewise essential because sample size is critical for both population studies and structure surveys, and also in searching for rare objects. An 8m-class telescope is essential because normal galaxies at $z>1$ are very faint (the median redshift of the galaxy population only reaches $z=1$ for $K=21$ ).

Features of the AUSTRALIS design include: (i) spectral range: 0.5-1.8 microns (V to $\mathrm{H}$ bands); (ii) field of view: 20 arcmin diameter (VLT Nasmyth); (iii) feed mode 1: up to 400 individual 1.5 arcsec fibres; (iv) feed mode 2: integral field unit, FoV 9-18 arcsec, resolution 0.25-0.5 arcsec; (v) resolving power: $\mathrm{R} \approx 4000$, permitting digital $\mathrm{OH}$ sky-suppression; (vi) detectors: six Rockwell $1024^{2} \mathrm{HgCdTe}$ devices plus two $2048^{2}$ CCDs.

A detailed performance model suggests that feasible projects for such a spectrograph on the VLT would include a redshift survey of $10^{4} \mathrm{~K}<21$ galaxies $(50 \%$ at $z>1)$ in 10 nights and a study of the evolution of the star-formation rate and Tully-Fisher relation via $\mathrm{H} \alpha$ fluxes and linewidths down to $100 \mathrm{~km} \mathrm{~s}^{-1}$ for 800 galaxies over $z=0.6-1.7$ in 4 nights.

Such capabilities open up new horizons for studying the universe at high redshifts, and will ensure that wide-field, multi-object spectroscopy stays at the forefront of observational cosmology over the next decade and beyond.

\section{References}

Bellanger C., de Lapparent V., Arnouts S., et al., 1994, A\&AS, 110, 159

Bender R., Ziegler B., Bruzual G., 1996, ApJ, accepted

Carlberg R., Yee H., Ellingson E., et al., 1996, ApJ, 462, 32

Colless M.M., Dunn A.M., 1996, ApJ, 458, 435

Cowie L.L., Songaila A., Hu E.M., Cohen J.G., 1996, ApJ, submitted

Efstathiou G., Ellis R.S., Peterson B.A., 1988, MNRAS, 232, 431

Ellis R.S., Colless M.M., Broadhurst T., Heyl J., Glazebrook K., 1996, MNRAS, 280, 235

Heyl J.S., Colless M.M., Ellis R.S., Broadhurst T.J., 1996, MNRAS, submitted

Lilly S.J., Tresse L., Hammer F., Crampton D., Le Fevre O., 1995, ApJ, 455, 108

Loveday J., Peterson B.A., Efstathiou G., Maddox S.J., 1992, ApJ, 390, 338

Rix H.-W., Guhathakurta P., Colless M.M., Ing K., 1996, MNRAS, submitted

Santiago B.X., Strauss M.A., Lahav O., et al., ApJ, 446, 457

Shectman S.A., Landy S.D., Oemler A., et al., 1996, ApJ, accepted 
Sebring T.A., Adams M., Ramsey L.W., 1995, BAAS, 187, \#121.03

Simard L., Pritchet C.J., 1996, ApJL, submitted

Steidel C., Giavalisco M., Pettini M., Dickinson M., Adelburger K., 1996, ApJ, 462, L17

van Dokkum P.G., Franx M., 1996, MNRAS, accepted

Vogt N.P., Forbes D.A., Phillips A.C., et al., 1996, ApJL, accepted 